\title{
Utilizing Geographic Information Systems for Condition-Based Maintenance on the Energy Distribution Grid
}

\author{
Philipp zur Heiden \\ Paderborn University \\ philipp.zur.heiden@uni-paderborn.de \\ Jennifer Priefer \\ Paderborn University \\ jennifer.priefer@uni-paderborn.de \\ Daniel Beverungen \\ Paderborn University \\ daniel.beverungen@uni-paderborn.de
}

\begin{abstract}
The energy distribution grid is a critical infrastructure challenged with shifting requirements induced by the skyrocketing importance of green energy. Particularly, legacy assets-such as mediumvoltage switchgear cabinets and circuit breakersneed to be maintained to prevent energy outages and reduce resource consumption. While related research has abundantly presented algorithms for conditionbased maintenance, no design knowledge is available to prescribe how an information system for this purpose ought to be designed. In a design science research project, we develop an information system for condition-based maintenance of legacy assets in the medium voltage distribution grid that utilizes geospatial data. Our design integrates Enterprise Resource Planning (ERP) functionality with Geographic Information Systems (GIS) and a Machine Learning System (MLS) for predicting outages. We demonstrate a current proof-of-concept and conclude by presenting a set of theoretical hypotheses that can guide the evaluation once the system is available.
\end{abstract}

\section{Introduction}

The energy distribution grid is bound to adapt to multiple transformational factors to increasingly focus on sustainability. First, the distribution grid has to transition from traditional, centralized energy production to a more decentralized production and consumption of energy due to renewable energy generation [1]. Second, the political decision for a turnaround to favor green energy [2] forces the distribution grid to become bi-directional instead of only providing one-way energy flows. Third, electric vehicles increase the overall demand for electric energy [3]. These challenges and developments strain legacy assets in the distribution grid, requiring a higher amount of status monitoring and more sophisticated maintenance strategies. Legacy assets, e.g., switchgear cabinets and circuit breakers, are supposed to reach a lifetime of more than 20 years and most of the assets used in the field have been implemented while the current trends were unforeseeable.

To solve these challenging problems, distribution grid operators must keep their assets in good condition despite increasing expectations, based on improving their maintenance strategies. Condition-based maintenance - a maintenance strategy that is based on condition data analysis to predict upcoming failures and schedule maintenance activities [4] - has demonstrated first promising research results regarding critical assets in the distribution grid [5-7]. However, beyond first insights on the applicability of algorithms to predict outages, information systems that enable full condition-based maintenance strategies are yet to be designed.

Location analytics and geographic information systems (GIS), an important set of concepts gaining increased attention in different industries, can help to overcome the stated challenges and improve maintenance strategies on the distribution grid. Surprisingly, GIS and location analytics have not gained much attention in the Information Systems (IS) discipline, apart from applications in location-based services (LBS) [8, 9]. The data gathered in these systems - including an entity's geographic positionare processed and analyzed by filtering, selecting, compiling, or creating valuable information [10]. Individual service consumption and the overall value of service are optimized for LBS [10,11], which is documented by a wide range of theories and IT artifacts related to LBS. However, most of these services use geospatial data of moving objects, e.g., people and devices, with common examples including rental services, mobile advertising, and turn-by-turn navigation. Research on utilizing geospatial data of immobile objects, such as critical assets of the distribution grid, is scarce.

In this research paper, we address key challenges of the energy distribution grid with the following research question: How should a system for conditionbased maintenance on the distribution grid, utilizing 
geospatial data of immobile objects with location analytics and GIS for outage prediction, be designed? We align with the design science research paradigm [12] and follow the first steps of the design science research model as proposed by Peffers et al. [13].

With our results, we provide an instantiated system for condition-based maintenance of assets on the energy grid. Our contribution can foster new research in IS and GIS-related research. We show how to combine data analysis and geographic analysis of immobile objects like electric substations to build efficient and effective systems for condition monitoring and conceptualize how this type of service depends on GIS for its central value, bridging the gap between research on GIS and other IS research.

\section{Theoretical background}

\subsection{Maintenance strategies}

Maintenance as of checking and repairing industrial assets has drastically changed over the last years due to an enormous amount of data available from different sources. Reactive maintenance, preventive maintenance, and condition-based maintenance [4] have established as core maintenance strategies. Reactive maintenance describes a strategy of operating assets until a malfunction or defect occurs to then quickly repairing the asset. This strategy inherently neglects scheduled inspections and, thus, utilizes an asset's maximum lifespan, minimizing spare parts costs at the expense of increased downtimes [4].

Preventive maintenance strategies aim to inspect and replace components with spare parts once default thresholds are exceeded [4]. Preventive maintenance can prevent defects, but might also provoke replacements prior to their end of lifetime [4]. Thus, preventive maintenance strategies tend to be more expensive but more failure-proof compared to reactive maintenance strategies.

Assuming that failures do not always occur after fixed time intervals but can occur at random times [14], condition-based maintenance has established as an innovative maintenance strategy. Condition-based maintenance [15], also named as predictive maintenance, refers to reducing downtimes and failures by permanently monitoring condition changes through advanced sensor and signal processing [15]. Systems for supervisory control and data acquisitions (SCADA) can aggregate the sensor data to detect the current conditions, including data from other applications, e.g., ERP systems [16]. Condition-based maintenance heavily relies on the use of statistical models and machine learning (ML) methods to detect critical condition changes based on field data. ML aims to predict answers to yes or no questions by using (supervised) classification based on a training dataset and has already been found useful for the energy sector, e.g., by integrating historical electrical grid data into ML prediction models [17]. In theory, condition-based maintenance can ultimately avoid downtimes and still utilize assets and components to their end of lifetime [4]. This can help minimizing costs for maintenance as components can be used just before they break down and downtimes and related costs are avoided. In the aircraft sector conditionbased maintenance has been found to reduce maintenance costs of about 80 percent [18]. By 2020, more than 800 million smart meters are expected to be installed worldwide [19], but until now large parts of the Medium Voltage (MV) energy distribution grid are not smart yet [20]. Though, condition-based maintenance is already applied in some areas of the distribution grid, e.g., for wind turbines [7] and to identify factors impacting the reliability of transformers [21]. However, the extant research focuses on developing ML and data analysis models, while not conceptualizing a full system. Further, existing systems do consider neither geospatial data, nor location analytics, both of which could render important benefits.

\subsection{Location analytics and GIS}

The rise of digital technology and devices allows for generating an enormous amount of geospatial data. To analyze these geospatial features, location analytics has established to be a valuable concept, referring to "contemporary concept of using specialized spatial analysis techniques to understand spatial arrangements, patterns, groupings and relationships in geographically referenced phenomena" [22, p. 1]. Location analytics is mostly used to support decisionmaking in complex contexts [22], often supported by Geographic information systems (GIS) as a class of information systems. Dueker and Kjerne [23] establish GIS as systems of hardware, software, data, people, organizations, and institutional arrangements, which enable collecting, storing, analyzing, and disseminating information about areas of the earth. GIS allows users to measure and represent geographic phenomena [24] and provides a diverse set of methods to work with geospatial data. Thus, GIS enable users to integrate location as part of an information system [25].

In general, location analysis and GIS are interdisciplinary for they use methods and techniques from many disciplines, e.g., geography, cartography, computer science, spatial information science, 
information systems, operations research, statistics, economics, and business [26]. With the help of GIS, experts can accesses geospatial and attribute information, analyze it, and produce outputs that can influence business processes [25, 26]. Results include heat maps to visualize hot and cold spots or map representations of statistical method application, such as clustering [22].

GIS are significant for designing information systems because of their deep connections with decision support systems, marketing and retail systems, analytics, and big data [22, 25]. Surprisingly, though GIS offer a geospatial dimension for business decision making and can improve the efficiency and effectiveness of solutions [25], IS research has not given much attention to GIS yet [26]. Farkas et al. [25] notice that GIS have the potential to improve the efficiency of enterprise and transaction systems, support middle management, and inform corporate leaders on aspects of strategic visioning and decision making. These aspects would generate numerous opportunities for IS research, such as improving applications through enhancing their accuracy, efficiency, knowledge, and intelligence [25].

IS research mainly uses GIS for spatial analysis [27-29], public services [30], and decision support [22, 31-33]. Until now, the utility of GIS has yet to be discussed and GIS have rarely been developed in design science projects.

\section{Research method}

The goal of our research is to design a system based on analytical and geospatial data (positions of electric sub-stations and area-related data, e.g., weather data, vegetation, animal population), providing improved maintenance service of assets crucial for the distribution grid to ultimately reduce failures and downtimes. Our solution classifies as an IT artifact, and, as such, must be designed using design science as a central research paradigm in IS [12].
Design science is focused on the design of IT artifacts [12], i.e., constructs, models, methods, and instantiations [34]. Design science pursues a dual mission [35] of (1) yielding actionable and useful IT artifacts and (2) providing IS design theories. These IS design theories prescribe how specific classes of IT artifacts ought to be designed to solve classes of problems. Thus, the research paradigm of design science refers to "learning through building" [36]. For designing IT artifacts, the duality of form and context in design science research must be considered. IT artifacts as human-made artifacts embody the form, while the context - the environment of the IT artifact - defines the boundaries [12].

In our research approach, we apply the design science research methodology proposed by Peffers et al. [13], since it provides useful and accepted guidelines for developing design knowledge. We set out to adapt the research process covering six steps (Figure 1) to design an instantiation of a system for condition-based maintenance of the distribution grid. We choose a problem-centered initiation for our design process because we investigate the current problems of distribution grid operators and their central components. Therefore, we aim to enable grid operators to schedule maintenance activities according to the predicted remaining lifetime to ultimately reduce downtimes. The design and development of our IT artifact is based on interviews and previously derived requirements [37]. As such, we design a holistic system for condition-based maintenance on the distribution grid using GIS and develop a set of structural and behavioral models. We demonstrate our system, focusing on the GIS as a central system for presenting data and providing user interactions, using sample data.

Since a naturalistic evaluation would require a full-scale implementation of the system and an integration of its components, as well as condition detecting sensors implemented in a sufficient number of critical assets, evaluating our design is beyond our

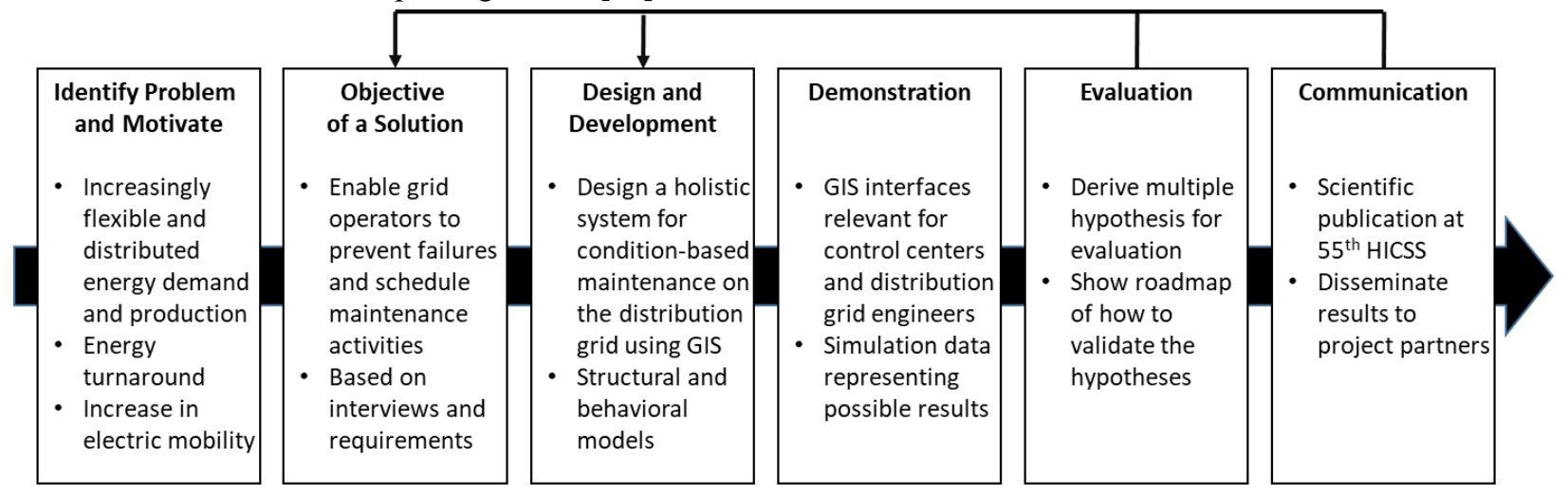

Figure 1. Instantiated design science research process, adapted from Peffers et al. [13]. 
current state of research. While we are involved in a consortium research project to develop a full-scale system with grid operators and component manufacturers, we must postpone the evaluation until a viable software prototype is available and deployed to the field, enabling us to compile a sound amount of field data to evaluate the system. To prepare the evaluation conceptually, however, we present a set of theoretical hypotheses that can guide the evaluation, once the system is available.

\section{Problem and objectives of a solution}

We embed our study in the context of the distribution grid and its operators on a MV level in central Europe. The MV grid is energized by high voltage providers - power plants and high voltage distribution grids. The grid operators' customers are mostly municipal utilities - distribution grid operators on the MV or low voltage (LV) level-and large industrial companies. Instead, private households and smaller industries buy their energy from municipal utility providers. The main assets of MV distribution grid operators are scattered around the operator's area, gathered in electrical distribution sub-stations. The number of sub-stations depends on the size of the distribution grid, mostly ranging from 5,000 to 10,000 sub-stations. These sub-stations contain different valuable and expensive assets, e.g., transformers, switchgear cabinets, and circuit breakers, all of which are subject to maintenance. Actually, operators usually implement a broad spectrum of maintenance approaches simultaneously, including reactive (conducting repairs after failures have occurred while no maintenance is performed between error), reliability-centered (risk-based assessment of consequences of possible failures [38]), and preventive maintenance (maintaining assets subject to pre-defined time intervals [4]).

From an organizational perspective, there are four roles associated with maintenance and repair services on the MV distribution grid. First, the control center agent is the first point of contact to investigate problems and disturbances of the distribution grid. It is also responsible for continuously monitoring the equipment. The control center agent schedules repairs and regular maintenance for senior distribution grid engineers and their mechanics. The senior distribution grid engineer manages a defined part of the distribution grid and knows stationary assets, whereas the distribution grid mechanics mostly fix and maintain the assets. Distribution grid planners monitor the condition of the distribution grid, while they also improve and extend the distribution grid. Distribution grid operators use different types of information systems for their work: Enterprise resource planning (ERP) systems for managing various resources (i.e., assets), GIS for planning and visualizing the distribution grid, and several other systems for data and information management.

However, with the rise of green energy and a focus on sustainable energy management, many of the legacy assets in the distribution grid are subjected to increasing strain. First, the production of and the demand for energy become more flexible and decentralized, promoted by the energy turnaround and an overall call for renewable energies [1, 2]. Renewable energies include photovoltaic cells, biogas plants, and wind power, which are often operated by private households or industrial companies. Second, the increasing use of distributed energy resources [39], electric vehicles, and electric mobility boost the demand for energy [3], while EVs might also be used for storing surplus energy temporarily [40]. Thus, the distribution grid needs to change from a previously unidirectional network for energy transportation to a bidirectional and decentralized network. Due to higher load variations in a bidirectional network, critical assets in the distribution grid come under pressure, while distribution grid operators struggle to maintain their part of the distribution grid [41].

Legacy components in the energy grid need to be made fit for the requirements imposed by the rise of green energy, since outages of critical assets will disrupt the energy supply and, ultimately, impede industry and consumers. As nearly all of society's modern services rely on energy - data-driven services based on computing resources are particularly energydependent-blackouts and energy shortages are disruptive. Consequences for, e.g., medical services (hospitals) and food services (lack of cooling) would be particularly severe.

The central objective of the system to be designed is to perform reliable and affordable maintenance for MV distribution grid operators to ultimately reduce downtimes. In a preceding study [37], we interviewed six distribution grid operator companies regarding their ideas and requirements for future solutions to perform maintenance. Aggregating their problems and ideas, we derived four requirements from these interviews informing the objectives of a solution.

(R-1) Condition-based maintenance: Distribution grid operators need to detect errors and information about malfunctioning assets pre-emptively and automatically, based on condition monitoring. They cannot wait for assets to fail but have to pre-emptively repair them applying a condition-based (predictive) maintenance strategy [4]. Condition-based maintenance is a promising approach that was successfully used in related scenarios $[6,7,14]$. As the 
ML algorithms' output, operators need failure probabilities and a time-to-failure computed for each critical asset. We continue to bundle these outputs as prognostics, consisting of availability (failure probability) and prognosis (estimated time-to-failure), according to ISO 13372 [15].

$(R-2)$ Condition monitoring: Permanent condition monitoring [15] is a foundation of condition-based maintenance. By collecting data from multiple sensors-e.g., voltage sensors, thermal sensors, camera sensors, air quality sensors - the condition of critical assets is monitored in real-time.

(R-3) Integrated suite of applications: System integration and reduced redundancies are another objective. Currently, information systems used by distribution grid operators are not (well) integrated. A suitable information systems architecture needs to include an ERP system, a GIS, and a monitoring system. Processes and data management need to be integrated to guarantee a flawless information flow.

(R-4) Knowledge base: Distribution grid operators need to document errors to build up knowledge. Some operators do not record and account errors yet and remain unable to improve their service. Our solution needs to report historical data and make them available for subsequent analysis.

\section{Results}

\subsection{System for condition-based maintenance}

We build upon the requirements $\mathrm{R}-1$ to $\mathrm{R}-4$ as objectives of our solution and present four services to be provided with our system, along with an information systems architecture, a data model, and process models. For our kernel theory, we use condition-based maintenance as a strategy to maintain critical assets [4, 15]. Our system is designed to function as an internal service for the distribution grid operator, though it could be outsourced to an external service contractor.

The three core services are divided by their time reference-analyzing the past, present, and future status of the distribution grid. Service order management as a fourth service completes our service portfolio. First, a reporting service analyzes historical condition data of the critical assets of the distribution grid to deliver valuable insights into cost analysis, grid planning, and strategic decisions. This service aims to analyze the past conditions in the distribution grid. It can apply different GIS-related capabilities to deliver insights relevant for planning and strategic decisions. The second service, condition monitoring, provides insights into the current state and condition of the assets (R-2). Third, prognostics analyzes the current and historic conditions combined with external data to predict the probability of failure and the time-tofailure (R-1). External data mainly include weather data, which were found to improve prediction models for electric grid outage and asset management [42]. In cases of disturbances or unexpected conditions during condition monitoring and prognostics, the Machine Learning System (MLS) automatically generates maintenance notifications for the affected assets. The control center agent can use these maintenance notifications to trigger service orders, which serve as a starting point for repair and maintenance in the service order management service. Additionally, service order management can be triggered manually by external messages about malfunctions (mostly by telephone).

The designed system is heavily based on information and communication technology. MLS, GIS, and ERP are commonly used by distribution grid operators and thus, are the three central information systems we integrate in our designed system (R-3). The MLS is connected to the different sensors equipped in the assets of the distribution grid (e.g., vibration sensors, temperature sensors, camera sensors, voltage sensors). It is responsible for data acquisition and data processing. To include external data, MLS can access data from an external weather system and can access equipment data and previous service order data from the ERP. In critical cases of assets, MLS can automatically create maintenance notifications stored in the ERP. As the focus of this paper is the design of the overall system, we refrain from explaining the technical implementation and algorithms related to $\mathrm{ML}$ and artificial intelligence. MLS is a system to be newly developed as there are no fitting solutions available.

The ERP system provides standardized processes. In our service system, the ERP system mainly revolves around providing data for the different assets and for documentation and tracing of maintenance notifications and service orders. For integration purposes defined in R-3, the ERP system provides interfaces for the data access (used by MLS and GIS) and creation of maintenance notification (used by MLS). Established ERP systems for maintenancerelated services-we used SAP Enterprise Asset Management (EAM) - provide a variety of interfaces.

The GIS's purpose is to provide a user interface, geographic analysis methods, and visualization of the distribution grid and its condition. Therefore, it accesses interfaces of both MLS and ERP, as well as external systems for weather data and geospatial data services. It offers energy suppliers numerous benefits for network data acquisition, planning, and keeping an overview of the entire network. Within the digital 


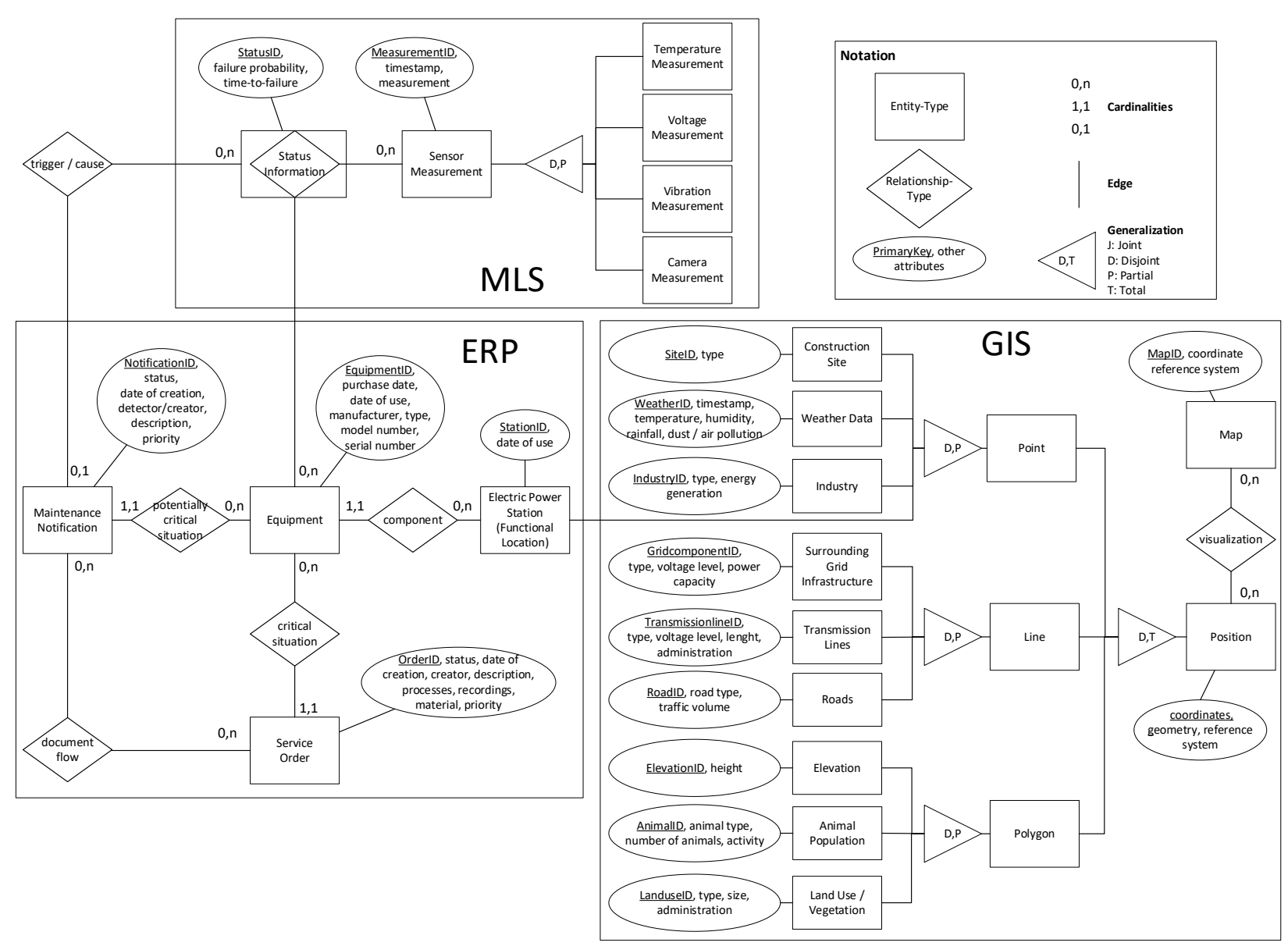

Figure 2. Entity-relationship model, representing the underlying data structures.

maps provided by a GIS, it is possible to differentiate the acquired data to the voltage levels: High Voltage (HV), MV, LV, lightning, and telecommunications. Power suppliers also have to possibility to map energy supply chains from centralized or decentralized power generation to the consumer as well as network connection or disconnection status information within a GIS. Due to knowing the position of grid components, it is possible to navigate to components in case of failure or to simulate failure scenarios such as affected areas or households in case of component downtime. For planning new network infrastructures, our GIS represents a sophisticated tool for spatial decision support. GIS allow spatial planning of new network components in a topological or geographical manner and provide the opportunity to consider environmental aspects like weather data and geospatial data services. Thus, a GIS can help to combine contextual data and display these data as either points, lines, or polygons on the digital map. Examples for contextual data applying to our services include animal populations (risk of causing short circuits), land use and vegetation (polluting electrical contacts), and transmission lines. Further, our GIS can visualize sensor data or failure probabilities as well as maintenance notifications according to substations and components and reveal problem areas in a visual way. We used Esri's ArcGIS Pro, a system providing GIS functions necessary for our implementation.

Next, we design a data model as an entityrelationship model [43] to display the data structures on which our system is based (cf. Figure 2). The tripartite structure reveals a close connection between GIS, MLS, and ERP, relating to the systems and required by R-3. For ERP and GIS, we did not include additional data compared to existing modeling approaches. However, entities of the MLS are new and relate to common equipment and maintenance notifications in SAP EAM. The different position entities of the types point, line, and polygon show the contextual data the GIS contributes to our conditionbased maintenance service system. Without this information, automatically analyzing and manually inspecting critical scenarios would not be possible, limiting the value-in-use provided by our service.

Further, we design business processes for our services with BPMN 2.0. With our process models and data model, we display the central structure and 


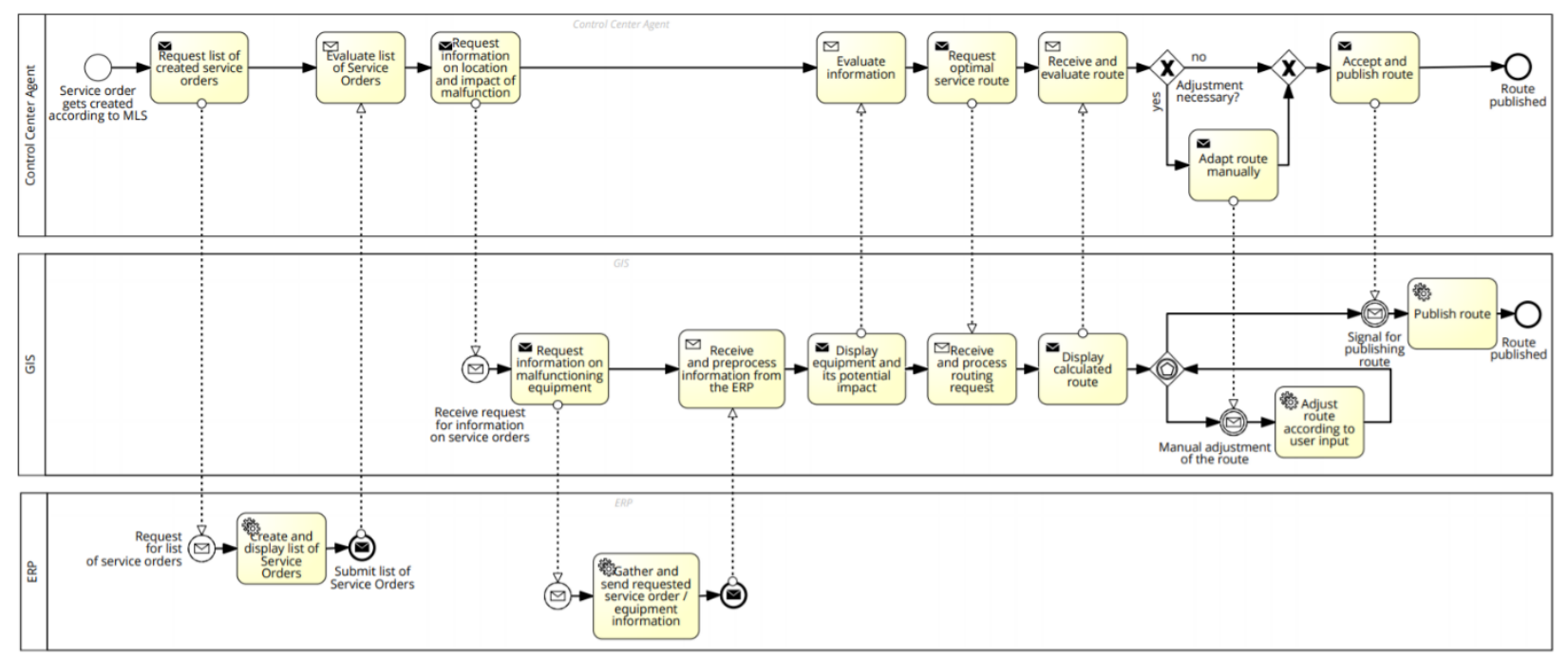

Figure 3. Model of an extract from the service order management process (BPMN).

behavior of our service system. As condition monitoring and prognostics both output maintenance notifications even if they consider different data for their analysis, we combine them into one process to reduce redundancies (R-3). The process involves an automatic calculation of the current or a prediction of the future condition of the distribution grid in MLS. In error cases, the MLS checks for existing maintenance notifications stored in the ERP and may create new ones. Next, as the only human actor in this process, the control center agent views a new maintenance notification in the ERP, its data in the MLS, and its geospatial data in the GIS. The control center agent then decides if a maintenance notification should be closed or creates a service order based on this maintenance notification to trigger maintenance activities on the distribution gird.

The process for the service order management service involves the manual service order creation in cases of manually reported malfunctions, preparation of the service order, and execution of the service order. The preparation is done by the control center agent supported by all three information systems: ERP for core data of assets, MLS for insights and condition data, and GIS for route planning and geographical insights. A central except for the process model in BPMN is visualized in Figure 3. It sketches the value the GIS is able to co-create in the service order management process. A senior distribution grid engineer and additional distribution grid mechanics execute the service order by viewing information displayed in the three information systems, ordering material, driving to the substation, and completing necessary repair and maintenance tasks. Finally, the senior distribution grid engineer documents the material and works on the service order, ending service order management. The documentation within this process is mostly automated, documenting the insights gained from the different systems and storing them in the ERP system (R-4).

Regarding R-4, with reporting, grid planning engineers can access historical data from different information systems for grid planning, cost-based calculations, modernization activities, and spatial analyses. The process is designed flexibly to enable other data to be considered for strategic decisions based on the grid's historical condition and data.

\subsection{Demonstration}

To demonstrate our IT artifact, we focus on the presentation of the maintenance-specific data using GIS. As our solution is not yet implemented and we do not target the ML algorithms for detecting failures and demands for maintenance, we rely on simulation data of detected failures. We map the distribution grids' assets with the help of Esri's ArcGIS which imports data from the MLS. Using GIS enables us to visualize the current status of grid assets and their maintenance tasks and builds the basis for a quantity of analyses. For example, grid planners can analyze which areas of the distribution grid suffer from frequent failures and determine if this could be related to external influences. Therefore, different data types need to be included in the GIS.

First, our system is able to present the current status of the grid assets by visualizing their probability of failure. These data are received directly from the MLS and is updated every few seconds. Figure 4 shows a sample of grid assets in Paderborn, Germany, being visualized in different colors and sizes according to their current probability of failure. By displaying the information on a map using GIS, the energy supplier 


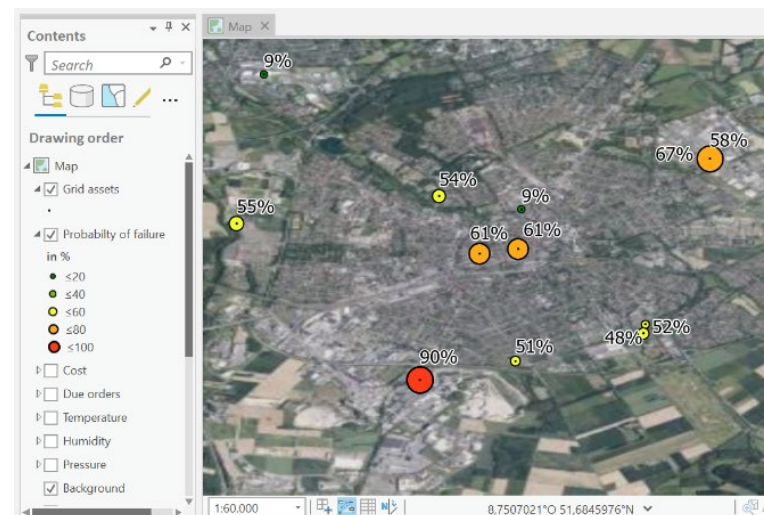

Figure 4. Visualization of failure probabilities of grid assets in ArcGIS.

can directly identify the areas in which outages are likely to occur and, for example, immediately route a grid engineer to maintain assets that stand in close proximity.

Second, through the interface between the SAP system and GIS, it is possible to display information on orders (due dates, costs resources) as map elements. This information is updated frequently and reveals spatial relationships, e.g. where appropriate routes or areas with upcoming maintenance activities. Figure 5 shows our sample dataset with information about maintenance due dates and costs according to the corresponding order from the SAP system.

In addition to the condition of the assets and the corresponding maintenance order, our IT artifact also enables the integration of environmental data comprising weather, elevation, animal population, and vegetation. The visualization of these surrounding conditions allows for grid planners to analyze spatial relations and dependencies in certain network areas.

\subsection{Evaluation}

Evaluation is a crucial step in design science research and can be done by applying different methods and techniques [12]. In our study, we designed an instantiation of a condition-based maintenance system to monitor and maintain healthy conditions of critical assets of the distribution grid. Our designed solution should be evaluated in two ways. First, a holistic evaluation should compare today's critical errors, blackout times, asset reliability, process efficiency, and maintenance costs to the situation after implementing our proposed service system. Such an evaluation shows the value, efficiency, and effectiveness of the designed solution. Second, functionality and usability should be tested with the system's customers: distribution grid engineers and control center agents. This second evaluation should detect neglected functions needed

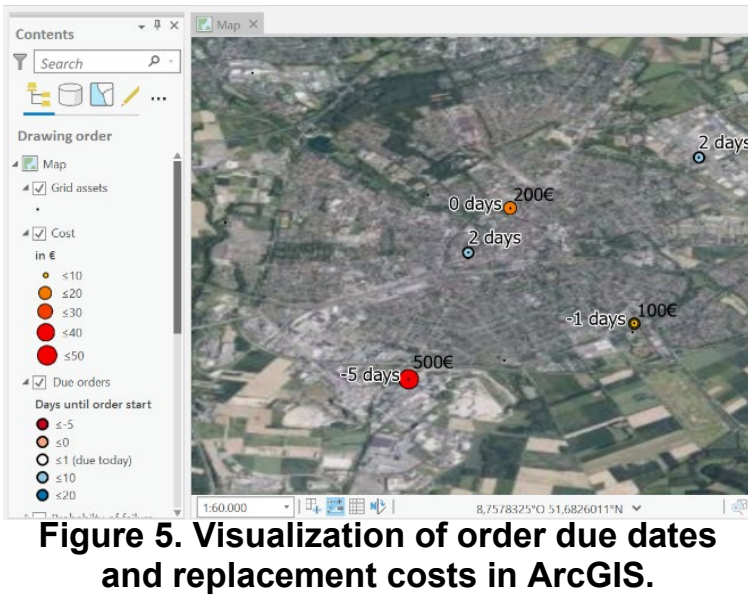

by the customers and might identify related problems of our system to improve our designed solution.

However, both of these evaluations require an implementation with sufficient historical condition data. All systems would have to be fully integrated (see R-3), control center agents and distribution grid engineers need to be trained, and, most importantly, a majority of the critical assets of the distribution grid needs to be equipped with sensors for different condition monitoring measurements. Until now, the distribution grid's critical assets lack sensors and functionality to transfer sensor data. Thus, an adequate and convincing evaluation of our designed system is not yet possible. We, therefore, need to postpone a naturalistic evaluation of our condition-based maintenance system until a software prototype is available and has been deployed to the field, resulting in proper field data to use in our evaluation.

Instead, we focus on conceptually preparing the evaluation by developing a set of theoretical hypotheses that can guide the evaluation, once the system is available.

(H-1) Fewer downtimes of critical assets: Our proposed system detects and reports malfunctions of assets pre-emptively and automatically, leading to early warnings to prevent failures fewer downtimes of critical assets. This hypothesis could be measured by the sum of downtimes of parts of the distribution grid in its current state (control group) and the same parts with our system in place, in which we would expect a significant reduction of downtimes.

$(H-2)$ Stretching regular maintenance intervals: As a permanent condition monitoring of critical assets by using data from multiple sensors provides greater knowledge about the current state of the different assets, the intervals of regular maintenance cycles can be stretched out. Some steps of this cyclic maintenance could even be avoided due to the condition being monitored. This hypothesis could be measured by the sum of invested maintenance hours per part for the 
distribution grid in its currents state (control group) and with the implementation and installation of our system for condition-based maintenance. We expect the hours of maintenance to be significantly lower when our proposed system is in place.

(H-3) Improved error cause detection: Causes in cases of failures, which our system cannot fully prevent, can be detected faster and more precise due to less redundancies in the underlying data, real-time condition monitoring, and visualization. Nevertheless, this increase in knowledge is hard to measure because failures occur at random times [14] and the cause and its identification might differ, requiring a large-scale field study to affirm this hypothesis.

\section{Discussion and conclusion}

The designed system for condition-based maintenance on the distribution grid and its critical assets can be generalized to a context outside of the distribution grid. Our design results can be valid for all cases of condition-based maintenance impacting geographically distributed assets. These assets can be either immobile, e.g., wind turbines, heating and aircondition systems, or mobile, e.g., cars. Both scenarios can benefit from including geospatial data of the assets made accessible by a GIS to improve result presentation and, overall, reduce downtimes and stretch maintenance intervals.

This paper contributes to several streams of research. We show that GIS can be a foundation for analyzing transaction data and location data for maintaining legacy components on the distribution grid and other critical infrastructures. While a similar system can be designed without GIS, we posit that using geospatial data will lead to generate superior value-in-use. Geospatial data on immobile entitiese.g., regarding the distribution grid-are often available, even if they have been considered rarely in condition-based maintenance systems.

Also, GIS are still an underexplored topic IS design [26], whereas our system considers a GIS as a central resource for value co-creation. Thus, we bridge the gap between IS and GIS, enabling researchers to examine the role and value of GIS for IS research further. In terms of advancing research on GIS, our instantiation of condition-based maintenance system for the distribution grid's critical assets serves as an example of a future GIS application that integrates with enterprise systems (including ERP systems) and with ML components that predict outages based on data obtained from assets deployed to the field.

Limitations of our work include the missing evaluation that completes the research and results in further information about the use of the IT artifact.
Further, the particular instantiation of the MLS is still in development. Additionally, the results of the interviews delivering the requirements for our system might differ depending on the country, concerning different distribution grids all over the world.

While a naturalistic evaluation is pending until an instantiated system provides field data, we presented a conceptual roadmap to guide the evaluation. Future research can build on our systems architecture, data model, and processes to implement their own condition-based maintenance systems on the distribution grid, utilizing geospatial data. Further, our results should be evaluated with interviews and case studies of other distribution grid operators to identify the willingness to pay and costs required to implement our system. Future research should also focus on different ML algorithms within such systems, evaluating the role of mapping and visualization using GIS and the consideration of usability with regard to a condition-based maintenance system based on our architecture and data model.

Acknowledgements: This paper was developed in the research project FLEMING, which is funded by the German Federal Ministry for Economic Affairs and Energy (BMWi), promotion sign 03E16012F.

\section{References}

[1] G. V. B. Kumar, R. K. Sarojini, K. Palanisamy, S. Padmanaban, and J. B. Holm-Nielsen, "Large Scale Renewable Energy Integration: Issues and Solutions," Energies, vol. 12, no. 10, p. 1996, 2019.

[2] O. Renn and J. P. Marshall, "Coal, nuclear and renewable energy policies in Germany: From the 1950 s to the "Energiewende"," Energy Policy, vol. 99, pp. 224-232, 2016.

[3] M. Mureddu, A. Facchini, A. Scala, G. Caldarelli, and A. Damiano, "A Complex Network Approach for the Estimation of the Energy Demand of Electric Mobility," Scientific reports, vol. 8, no. 268, 2018.

[4] R. K. Mobley, An introduction to predictive maintenance, 2nd ed. Amsterdam, New York: Butterworth-Heinemann, 2002.

[5] F. P. García Márquez, A. M. Tobias, J. M. Pinar Pérez, and M. Papaelias, "Condition monitoring of wind turbines: Techniques and methods," Renewable Energy, vol. 46, pp. 169-178, 2012.

[6] A. Goyal et al., "Asset health management using predictive and prescriptive analytics for the electric power grid," IBM J. Res. \& Dev., vol. 60, no. 1, 2016.

[7] M. Canizo, E. Onieva, A. Conde, S. Charramendieta, and S. Trujillo, "Real-time predictive maintenance for wind turbines using Big Data frameworks," IEEE ICPMH, pp. 70-77, 2017.

[8] A. Küpper, Location-Based Services. Fundamentals and Operation. Chichester, UK: John Wiley \& Sons, Ltd, 2005. 
[9] J. Raper, G. Gartner, H. Karimi, and C. Rizos, "A critical evaluation of location based services and their potential," Journal of Location Based Services, vol. 1, no. 1 , pp. 5-45, 2007.

[10] B. McKenna, T. Tuunanen, and L. A. Gardner, "Exploration of Location-Based Services Adoption," International Journal of E-Services and Mobile Applications, vol. 6, no. 1, pp. 1-22, 2014.

[11] B. Rao and L. Minakakis, "Evolution of mobile location-based services," Commun. ACM, vol. 46, no. 12, pp. 61-65, 2003.

[12] A. Hevner, S. T. March, J. Park, and S. Ram, "Design Science in Information Systems Research," MIS Quarterly, vol. 28, no. 1, pp. 75-106, 2004.

[13] K. Peffers, T. Tuunanen, M. A. Rothenberger, and S. Chatterjee, "A Design Science Research Methodology for Information Systems Research," Journal of MIS, vol. 24, no. 3, pp. 45-77, 2008.

[14] H. M. Hashemian, "State-of-the-Art Predictive Maintenance Techniques," IEEE Trans. Instrum. Meas., vol. 60, no. 1, pp. 226-236, 2011.

[15] ISO 13372, Condition monitoring and diagnostics of machines: Vocabulary: Beuth, 2012.

[16] P. Panfilov and A. Katona, "Building Predictive Maintenance Framework for Smart Environment Application Systems," Proceedings of the 29th DAAAM Symposium 2018, vol. 1, pp. 460-470.

[17] C. Rudin et al., "Machine learning for the Ney York City power grid," IEEE TPAMI, vol. 34, no. 2, pp. 328-345, 2011.

[18] M. Gerdes, D. Scholz, and D. Galar, "Effects of condition-based maintenance on costs caused by unscheduled maintenance of aircraft," Journal of Quality in Maintenance Engineering, 2016.

[19] Telefónica, Prognosis for the number of installed smart meters in 2020 (in German). [Online]. Available: https://de.statista.com/statistik/daten/ studie/298351/ (accessed: Aug. 24 2021).

[20] J. Heckel, "Smart substation and feeder automation for a SMART distribution grid," in 2009, pp. 1-4.

[21] H. de Faria, J. G. S. Costa, and J. L. M. Olivas, "A review of monitoring methods for predictive maintenance of electric power transformers based on dissolved gas analysis," Renewable and Sustainable Energy Reviews, vol. 46, pp. 201-209, 2015.

[22] J. B. Pick, O. Turetken, A. V. Deokar, and A. Sarkar, "Location analytics and decision support: Reflections on recent advancements, a research framework, and the path ahead," DSS, vol. 99, pp. 1-8, 2017.

[23] K. J. Dueker and D. Kjerne, Multipurpose Cadastre: Terms and Definitions: American Society of Photogrammetry and Remote Sensing, 1989.

[24] N. R. Chrisman, "What does 'GIS'mean?," Transactions in GIS, vol. 3, no. 2, pp. 175-186, 1999.

[25] D. Farkas, B. Hilton, J. Pick, H. Ramakrishna, A. Sarkar, and N. Shin, "A tutorial on geographic information systems: A ten-year update," Com. of the AIS, vol. 38, no. 1, pp. 190-234, 2016.

[26] J. B. Pick, "Geographic information systems: A tutorial and introduction," The Communications of the AIS, vol. 14, no. 1, pp. 307-331, 2004.
[27] H. Arslan and D. Farkas, "Using GIS to understand the relationship of community factors and police shootings in the United States: a First Look," Proceedings of the 22nd AMCIS, San Diego, 2016.

[28] A. Lestari, G. Rumantir, and N. Tapper, "A spatiotemporal analysis on the forest fire occurrence in central kalimantan, indonesia," Proceedings of the 2016 PACIS, 2016.

[29] P. Keenan, P. Carroll, H. Conway, and S. Lee, "Spatial modelling of climate change in Irish Forestry," Proceedings of the 23rd AMCIS, Boston, 2017.

[30] O. Alrwais, B. Hilton, T. Horan, and T. Bechor, "An Organizational Perspective On GIS Payoffs for the Public Sector: Is Usage the Missing Link?," Proceedings of the 22nd AMCIS, San Diego, 2016.

[31] A. R. Albina, "Assessing the Impact of a GIS for Improving Novice Crisis Decision-Making," Proceedings of the 25th AMCIS, Cancun, 2019.

[32] M. d. Castro Neto, M. Nascimento, P. Sarmento, S. Ribeiro, T. Rodrigues, and M. Painho, "Implementation of a dashboard for security forces data visualization," CAPSI 2018 Proceedings, 2018.

[33] W. Suksa-Ngiam, J. Mbugua, and S. Chatterjee, "A GIS decision support system for crop cultivation," Proceedings of the 22nd AMCIS, San Diego, 2016.

[34] S. T. March and G. F. Smith, "Design and natural science research on information technology," DSS, vol. 15, no. 4, pp. 251-266, 1995.

[35] M. K. Sein, O. Henfridsson, S. Purao, M. Rossi, and R. Lindgren, "Action Design Research," MIS Quarterly, vol. 35, no. 1, pp. 37-56, 2011.

[36] B. Kuechler and V. Vaishnavi, "On theory development in design science research: anatomy of a research project," European Journal of Information Systems, vol. 17, no. 5, pp. 489-504, 2008.

[37] P. zur Heiden and J. Müller, "Transitioning to Condition-Based Maintenance on the Distribution Grid: Deriving Design Principles form a Qualitative Study," 16th International Congress on Wirtschaftsinformatik, Community Workshop, 2021.

[38] J. Moubray, Reliability-centered maintenance, 2nd ed. New York: Industrial Press, 1997.

[39] D. Pudjianto, C. Ramsay, and G. Strbac, "Virtual power plant and system integration of distributed energy resources," IET Renew. Power Gener., vol. 1, no. 1, pp. 10-16, 2007.

[40] J. B. Goodenough, "Electrochemical energy storage in a sustainable modern society," Energy Environ. Sci., vol. 7, no. 1, pp. 14-18, 2014.

[41] M. W. Hoffmann et al., "Integration of Novel Sensors and Machine Learning for Predictive Maintenance in Medium Voltage Switchgear to Enable the Energy and Mobility Revolutions," Sensors, vol. 20, no. 7, 2020.

[42] M. Kezunovic, Z. Obradovic, T. Djokic, and S. Roychoudhury, "Systematic Framework for Integration of Weather Data into Prediction Models for the Electric Grid Outage and Asset Management Applications," Proceedings of the 51st HICSS, 2018.

[43] P. P.-S. Chen, "The entity-relationship modeltoward a unified view of data," ACM transactions on database systems, vol. 1, no. 1, pp. 9-36, 1976. 